\title{
All solenoids of piecewise smooth maps are period doubling
}

\author{
by
}

\author{
Lluís A ls e dà (Barcelona), Víctor J i mén ez L ó pez (Murcia) and \\ L'ubomír S no ha (Banská Bystrica)
}

\begin{abstract}
We show that piecewise smooth maps with a finite number of pieces of monotonicity and nowhere vanishing Lipschitz continuous derivative can have only period doubling solenoids. The proof is based on the fact that if $p_{1}<\ldots<p_{n}$ is a periodic orbit of a continuous map $f$ then there is a union set $\left\{q_{1}, \ldots, q_{n-1}\right\}$ of some periodic orbits of $f$ such that $p_{i}<q_{i}<p_{i+1}$ for any $i$.
\end{abstract}

1. Introduction. Solenoids are important in one-dimensional discrete dynamical systems because they play a key role in the description of the asymptotic behaviour of a large class of maps from the family $C(I)$ of continuous maps from a real compact interval $I$ into itself. Namely, if $f \in C(I)$ is piecewise strictly monotone and smooth enough then there is a residual set $R$ such that for any $x \in R$ the set of limit points of the sequence $\left(f^{n}(x)\right)_{n=0}^{\infty}$ is either a periodic orbit, a finite union of closed intervals or a solenoid. Regarding this, the reader may for example wish to check [12], [10], to which we also refer for the basic background and terminology. (To ease a first reading of the Introduction, all definitions related to solenoids and the description of their relevant properties are postponed until the beginning

1991 Mathematics Subject Classification: Primary 58F12, 58F20.

Key words and phrases: Markov graph, periodic point, piecewise smooth map with nowhere vanishing Lipschitz continuous derivative, piecewise linear map, solenoid.

Much of the work on this paper was done during the stays of the second author at the Universitat Autònoma de Barcelona and the Matej Bel University. The invitation and the support of these institutions is gratefully acknowledged. The authors thank J. Plesník and R. Nedela for bringing the book [7] to their attention.

The first author has been partially supported by the DGICYT grant number PB930860, the second one by the DGICYT grant numbers PB91-0575 and PB94-1159, and the third one by the Slovak Grant Agency, grant number 1/1470/94-96. We are indebted to an anonymous referee for suggesting a proof of the proposition in Section 2 simpler than ours. 
of Section 3.) In this paper we deal with the question of what structure a solenoid of a piecewise smooth map can have.

In the particular case of piecewise linear maps this problem was previously considered in [5]. There it is proved that piecewise linear maps have no period doubling solenoids. In order to get this result a well known property of this type of solenoids is used. More precisely, period doubling solenoids generate a sequence $\left(P_{n}\right)_{n=0}^{\infty}$ of periodic orbits of period $2^{n}$ with the following geometrical feature. For each $n \geq 0$ let $P_{n}: a_{1}<\ldots<a_{2^{n}}$ and $\bigcup_{k=0}^{n-1} P_{k}: b_{1}<\ldots<b_{2^{n}-1}$. Then $a_{i}<b_{i}<a_{i+1}$ for any $i=1, \ldots, 2^{n}-1$. Thus, in trying to show the nonexistence of larger classes of solenoids for piecewise linear maps, the question arises whether for any periodic orbit $P$ of a continuous map there exist some other periodic orbits such that their points and the points of $P$ interlace as above. We answer this question in the affirmative:

Theorem A. Let $f \in C(I)$ and let $P: p_{1}<\ldots<p_{n}$ be a periodic orbit of $f$ with period $n \geq 2$. Then there is a set $Q: q_{1}<\ldots<q_{n-1}$ such that $f(Q)=Q$ and $p_{i}<q_{i}<p_{i+1}$ for any $i=1, \ldots, n-1$.

REMARK 1. Notice that $f(Q)=Q$ implies that $Q$ is the union of some periodic orbits of $f$.

REMARK 2. Theorem A will follow as an easy consequence of the fact that Markov graphs of periodic orbits of $f$ always have pairwise disjoint cycles such that every vertex of the graph belongs to one of them (cf. the Proposition in Section 2). This could be considered as a step towards a better graph-theoretical description of Markov graphs of periodic orbits.

Using Theorem A and some ideas from [5] we will be able to extend the above-mentioned result from [5] to show the nonexistence of a larger class of weakly bounded solenoids for piecewise linear maps (here "weakly bounded" means that the solenoid admits a decreasing covering by periodic intervals whose corresponding periods $k_{n}$ satisfy $\liminf _{n \rightarrow \infty} k_{n+1} / k_{n}<\infty$; see Section 3 for a precise definition).

Theorem B. If $f \in C(I)$ is piecewise linear, then it has no weakly bounded solenoids.

After we had proved Theorems A and B (November 1994) we found a recent paper by M. Martens and C. Tresser [9] where they succeed in proving that piecewise linear maps have no solenoids. They follow a similar reasoning to ours and also prove Theorem A.

It turns out that combining the ideas from Theorem B with additional tools developed in [9] it is possible to prove a surprising fact (which is not mentioned in [9]) concerning the nature of solenoids of piecewise smooth maps (with a finite number of pieces of monotonicity) whose derivative is 
Lipschitz continuous and nowhere vanishing (the precise definition will be given in Section 3). It is known that there exist examples of such maps having period doubling solenoids and, moreover, with the corresponding smooth pieces in the class $C^{\infty}$ (see [5]). In connection with this, examples by Bobok and Kuchta [3] of an expanding countably piecewise linear map with a period doubling solenoid and by Misiurewicz [11] of a countably piecewise linear map with a period doubling solenoid of positive Lebesgue measure (compare with Lemma 5 in Section 3) are also worth noticing.

The solenoid in the example from [5] turns out to be of the only possible type, since we prove:

Theorem C. Let $f \in C(I)$ be a piecewise smooth map with nowhere vanishing Lipschitz continuous derivative. Then any solenoid of $f$ must be period doubling.

Since piecewise linear maps belong to the above class, Theorem $\mathrm{C}$ implies that piecewise linear maps can have only period doubling solenoids. But by Theorem B (or by [5]) they have no period doubling solenoids. In other words, the main result of [9] is now an immediate corollary of Theorems $\mathrm{C}$ and B:

Corollary D. If $f \in C(I)$ is piecewise linear, then it has no solenoids.

In [6] Kolyada proved that if $f \in C(I)$ is such that for some $c \in I$, $f(x)=\left(a_{1} x+b_{1}\right) /\left(c_{1} x+d_{1}\right)$ for $x \leq c$ and $f(x)=\left(a_{2} x+b_{2}\right) /\left(c_{2} x+d_{2}\right)$ for $x \geq c$ with $a_{i} d_{i}-b_{i} c_{i} \neq 0$ for $i=1,2$, then it has no period doubling solenoids. Denote the set of all such maps by $K_{2}(I)$ (the index 2 indicates that these maps consist of two pieces). Since $K_{2}(I)$ is included in the class of maps considered in Theorem C, from Kolyada's result we get:

Corollary E. If $f \in K_{2}(I)$, then it has no solenoids.

We conjecture that this result can be extended to similar maps with an arbitrary (finite) number of pieces of monotonicity.

REMARK 3. Notice that Theorem $\mathrm{C}$ implies, in particular, that for any positive integer $l$ the family of $l$-modal maps from $\operatorname{Lp}(I)$ cannot be full. After we had written a first version of this paper we learned about the recent paper [4] by R. Galeeva and S. van Strien where it is shown that somewhat different but related families of piecewise smooth maps cannot be full either.

2. Proof of Theorem A. A powerful tool to handle problems related to the combinatorial structure of periodic orbits is that of Markov graphs (see [1] for more details). Recall that if $V$ is a nonempty finite set and $E \subset V \times V$ we call $G=(V, E)$ a directed graph. Let $f \in C(I)$ and let $P$ 
be a set $a_{1}<\ldots<a_{n}$ of points from $I$ with $n \geq 2$. If $V=\left\{\left[a_{i}, a_{i+1}\right]\right.$ : $i=1, \ldots, n-1\}$ and we define $\left(\left[a_{i}, a_{i+1}\right],\left[a_{j}, a_{j+1}\right]\right) \in E$ if and only if $f\left(\left[a_{i}, a_{i+1}\right]\right) \supset\left[a_{j}, a_{j+1}\right]$, then the corresponding directed graph $G=(V, E)$ is called the Markov $f$-graph of $P$.

A directed graph $G=(V, E)$ is said to have a 1-difactor if there is a bijection $\Phi: V \rightarrow V$ such that $(x, \Phi(x)) \in E$ for any $x \in V$. Clearly, $G$ has a 1-difactor if and only if there exists a family of pairwise disjoint cycles in $G$ such that every vertex of $G$ belongs to one of the cycles.

We are going to show that Markov $f$-graphs of periodic orbits have a 1-difactor. This can be immediately shown by proving a stronger statement. In what follows, a finite set $P \subset I$ is said to be a strongly invariant set (of $f)$ if $f(P)=P$.

Proposition. Let $f \in C(I)$ and let $P \subset I$ be a strongly invariant set with Card $P \geq 2$. Then the Markov $f$-graph of $P$ has a 1-difactor.

Proof. Let $(V, E)$ be the Markov $f$-graph of $P$. In order to show that $(V, E)$ has a 1-difactor it suffices to check, according to the Marriage Lemma (see e.g. Corollary 1.1.4 of [7]), that for any $A \subset V$ the set of points $y \in V$ for which there exists $x \in A$ such that $(x, y) \in E$ has cardinality greater than or equal to the cardinality of $A$.

For simplicity assume $P=\{1, \ldots, n\}$. Define $I_{i}=[i, i+1]$ for $i=1, \ldots$, $n-1$. The union of the elements of $A$ is a union of finitely many pairwise disjoint intervals with endpoints in $P$. Consider one of these, $[i, i+j]$. Its image contains $I_{f(m)}$ for each $m=i, i+1, \ldots, i+j$ (except maybe one for which $f(m)$ is the largest), that is, $j$ of the intervals $I_{i}$. Since $f$ is a bijection on $P$, for another interval $\left[i^{\prime}, i^{\prime}+j^{\prime}\right]$ the corresponding intervals $I_{f\left(m^{\prime}\right)}$ are different. Therefore the total number of intervals $I_{r}$ in the image is at least as large as the cardinality of $A$, exactly what we wanted to prove.

We are now ready to prove Theorem A.

Proof of Theorem A. Put $I_{i}=\left[p_{i}, p_{i+1}\right], i=1, \ldots, n-1$. Let

$$
\Phi:\left\{I_{1}, \ldots, I_{n-1}\right\} \rightarrow\left\{I_{1}, \ldots, I_{n-1}\right\}
$$

be the bijection from the proof of the Proposition. Let $\sigma:\{1, \ldots, n-1\} \rightarrow$ $\{1, \ldots, n-1\}$ be defined by $\sigma(i)=j$ if $\Phi\left(I_{i}\right)=I_{j}$. Then we can decompose $\{1, \ldots, n-1\}$ into pairwise disjoint sets $N_{1}, \ldots, N_{k}$ with the following property. Set $n_{m}=$ Card $N_{m}, m=1, \ldots, k$. Then $\sigma\left(N_{m}\right)=N_{m}$ and, for any $i \in N_{m}, \sigma^{n_{m}}(i)=i$ while $\sigma^{j}(i) \neq i$ if $0<j<n_{m}$ (of course, $\sigma^{j}$ denotes the $j$ th iterate of $\sigma$ ). Fix an $i_{m} \in N_{m}$ for any $m$. By Corollary 1.2.8 of [1] there is $x_{m} \in I_{i_{m}}$ such that $f^{n_{m}}\left(x_{m}\right)=x_{m}$ and $f^{j}\left(x_{m}\right) \in I_{\sigma^{j}\left(i_{m}\right)}$ for any $j=0,1, \ldots, n_{m}-1$. Since $n_{m} \leq n-1$ we have $f^{j}\left(x_{m}\right) \notin P$ for any $j$ and so $f^{j}\left(x_{m}\right) \neq f^{i}\left(x_{m}\right)$ for $i, j \in\left\{0,1, \ldots, n_{m}-1\right\}$ with $i \neq j$. Hence, $x_{m}$ is a 
periodic point of $f$ of period $n_{m}$. Let $Q_{m}$ be the periodic orbit containing $x_{m}$. Then $Q=\bigcup_{m=1}^{k} Q_{m}$ is the strongly invariant set of $f$ we were looking for.

REMARK 4. Theorem A does not necessarily hold if $P$ is a strongly invariant set of $f$. To see this consider the map $f:[0,1] \rightarrow[0,1]$ defined by $f(x)=x^{2}$ and $P=\{0,1\}$. Then $P$ is a strongly invariant set of $f$ but $(0,1)$ does not contain any periodic points of $f$.

3. Proofs of Theorems B and C. Let us start with some notations and definitions.

If $A \subset I$ then Conv $A$ denotes the convex hull of $A$. Also, if $x, y \in I$, we shall write $[x ; y]$ to denote $\operatorname{Conv}\{x, y\}$. If $P$ is finite and we write $P=$ $\left\{a_{1}, \ldots, a_{n}\right\}$ then we always assume that $a_{1}<\ldots<a_{n}$.

In what follows, $D(I)$ will denote the set of piecewise smooth maps from $I$ into itself whose derivatives do not vanish. More exactly, $f \in D(I)$ if there are points $\min I=a_{0}<a_{1}<\ldots<a_{n}=\max I$ such that the restriction $f_{i}$ of $f$ to $\left[a_{i}, a_{i+1}\right]$ is $C^{1}$ and $f_{i}^{\prime}(x) \neq 0$ for any $x \in\left[a_{i}, a_{i+1}\right]$, $i=0,1, \ldots, n-1\left(f^{\prime}\left(a_{i}\right)\right.$ and $f^{\prime}\left(a_{i+1}\right)$ mean the corresponding one-sided derivatives). We always assume that the cardinality of $\left\{a_{0}, a_{1}, \ldots, a_{n}\right\}$ is the minimal possible satisfying the above properties and we denote such a set by $K(f)$. In particular, $f^{\prime}(a)$ exists for no $a \in K(f)$. The set of maps from $D(I)$ for which additionally every $f_{i}$ is linear (resp. every $f_{i}^{\prime}$ is Lipschitz continuous) will be denoted by $L(I)(\operatorname{resp} . \operatorname{Lp}(I))$. We emphasize that in the Introduction maps from $L(I)$ and $\operatorname{Lp}(I)$ were called piecewise linear maps and piecewise smooth maps with nowhere vanishing Lipschitz continuous derivative, respectively.

Let $f \in C(I)$ and let $J$ be a subinterval of $I$. If $J$ contains no asymptotically periodic points and $f^{n}(J) \cap f^{k}(J)=\emptyset$ for any $n>k \geq 0$ then it is called a wandering interval of $f$. The dynamics of maps from $C(I)$ may be substantially complicated due to the presence of wandering intervals but, fortunately, for a large class of "natural" maps (including all maps from $\operatorname{Lp}(I)$ - cf. [8], Theorem $\mathrm{A}^{\prime}$, or [10], pp. 285-286) wandering intervals cannot exist.

Let us next define the notion of a solenoid. Let $f \in C(I)$. We say that a sequence $\left(I_{i}\right)_{i=0}^{k-1}$ of closed subintervals of $I$ is periodic of period $k$ for $f$ if the intervals $I_{i}$ have pairwise disjoint interiors, $f\left(I_{i}\right) \subset I_{i+1}$ for any $i=$ $0,1, \ldots, k-2$ and $f\left(I_{k-1}\right) \subset I_{0}$. Sometimes we identify this sequence with the set of its terms and so we write $I_{i} \in\left(I_{i}\right)_{i=0}^{k-1}$ for any $i=0,1, \ldots, k-1$. We say that $A \subset I$ is a solenoid of $f$ if there exist a strictly increasing sequence $\left(k_{n}\right)_{n=1}^{\infty}$ of positive integers and periodic sequences $\mathcal{C}^{n}=\left(I_{i}^{n}\right)_{i=0}^{k_{n}-1}$ of period $k_{n}$ of closed intervals such that $\bigcup_{i=0}^{k_{n}-1} I_{i}^{n} \supset \bigcup_{i=0}^{k_{n+1}-1} I_{i}^{n+1}$ for any 
$n$ and $A=\bigcap_{n=1}^{\infty} \bigcup_{i=0}^{k_{n}-1} I_{i}^{n}$. The sequence $\left(\mathcal{C}^{n}\right)_{n=1}^{\infty}$ will be called a covering of $A$ of type $\left(k_{n}\right)_{n=1}^{\infty}$.

It is not difficult to show that for any $J \in \mathcal{C}^{n+1}$ there is $K \in \mathcal{C}^{n}$ such that $J \subset K$. Moreover, $k_{n}$ divides $k_{n+1}$ for any $n$ and each interval from $\mathcal{C}^{n}$ contains exactly $k_{n+1} / k_{n}$ intervals from $\mathcal{C}^{n+1}$. If a solenoid $A$ admits a covering of type $\left(k_{n}\right)_{n=1}^{\infty}$ with $\liminf _{n \rightarrow \infty} k_{n+1} / k_{n}<\infty$ then we say that $A$ is a weakly bounded solenoid of $f$. If in particular it admits a covering of type $\left(k_{n}\right)_{n=1}^{\infty}$ with $k_{n+1} / k_{n}=2$ for any $n$ large enough then we call $A$ a period doubling solenoid. In the opposite case we say that $A$ is non-period doubling.

Depending on the nature of $f$, solenoids may have some additional properties, which are next described in progressively more restrictive settings. After this we shall be able to prove Theorem B.

First of all it is easy to show that if $f \in C(I)$ and $A$ is a solenoid of $f$ then

$$
f(A) \subset A
$$

and

(2) $A$ does not contain any asymptotically periodic point of $f$.

Assume additionally that $f \in D(I)$. Then we say that a covering $\left(\mathcal{C}^{n}\right)_{n=1}^{\infty}$ of $A$ is strict if $K(f) \cap \bigcup_{J \in \mathcal{C}^{1}} J=K(f) \cap A$ and the endpoints of every interval from any $\mathcal{C}^{n}$ belong to $A$. From (2) we deduce in particular that

(3) if the covering is strict then the intervals from $\mathcal{C}^{n}$ are pairwise disjoint for any $n$.

Notice also that if $J \in \mathcal{C}^{n}$ and $J_{1}, \ldots, J_{l}$ are the intervals from $\mathcal{C}^{n+1}$ included in $J$ then

$$
J=\operatorname{Conv}\left(J_{1} \cup \ldots \cup J_{l}\right) \text { provided the covering is strict. }
$$

Observe further that each solenoid $A$ of $f \in D(I)$ admits a strict covering. In fact, if $\left(\mathcal{C}^{n}\right)_{n=1}^{\infty}$ is an arbitrary covering of $A$ with $\mathcal{C}^{n}=\left(I_{i}^{n}\right)_{i=0}^{k_{n}-1}$ then there exists $n_{0}$ large enough such that $K(f) \cap \bigcup_{i=0}^{k_{n_{0}}-1} I_{i}^{n_{0}}=K(f) \cap A$. Then it is easy to see that $\left(\left(\operatorname{Conv}\left(A \cap I_{i}^{n}\right)\right)_{i=0}^{k_{n}-1}\right)_{n=n_{0}}^{\infty}$ is a strict covering of $A$ (use (1)). Also, notice that if $\left(\mathcal{C}^{n}\right)_{n=1}^{\infty}$ and $\left(\mathcal{D}^{m}\right)_{m=1}^{\infty}$ are strict coverings of $A$ with $\mathcal{C}^{n}=\left(I_{i}^{n}\right)_{i=0}^{k_{n}-1}$ and $\mathcal{D}^{m}=\left(J_{j}^{m}\right)_{j=0}^{l_{m}-1}$ then $k_{n}<l_{m}$ (resp. $\left.k_{n}=l_{m}\right)$ implies $\bigcup_{i=0}^{k_{n}-1} I_{i}^{n} \supset \bigcup_{j=0}^{l_{m}-1} J_{j}^{m}$ (resp. $\left.\bigcup_{i=0}^{k_{n}-1} I_{i}^{n}=\bigcup_{j=0}^{l_{m}-1} J_{j}^{m}\right)$.

Assume now that a map $f \in D(I)$ has no wandering intervals (recall that this is for example the case if $f \in \operatorname{Lp}(I))$. If $\left(\mathcal{C}^{n}\right)_{n=1}^{\infty}$ is a covering of a solenoid $A$, it is easy to show (see [5]) that the lengths of the intervals from $\mathcal{C}^{n}$ tend uniformly to 0 as $n$ tends to $\infty$. This fact, together with the last comment from the above paragraph, clearly implies that for any solenoid $A$ 
of $f$ there exists a unique maximal strict covering $\left(\mathcal{B}^{n}\right)_{n=1}^{\infty}$ of $A$ such that each of the intervals from $\mathcal{B}^{n}$ contains at most one point from $K(f)$ (here "maximal" means that if $\left(\mathcal{D}^{m}\right)_{m=1}^{\infty}$ is another strict covering of $A$ with the same property then for any $m$ there is $n(m)$ such that $\left.\mathcal{D}^{m}=\mathcal{B}^{n(m)}\right)$. We call this covering the basic covering of $A$ and its type the type of $A$. Notice that, with this terminology, $A$ will be weakly bounded (resp. period doubling) if and only if the type $\left(k_{n}\right)_{n=1}^{\infty}$ of $A$ satisfies $\liminf _{n \rightarrow \infty} k_{n+1} / k_{n}<\infty$ (resp. $k_{n+1} / k_{n}=2$ for any $n$ large enough).

Of course, when defining the basic covering of a solenoid $A$ differentiability is not necessary, and then the construction also works for piecewise strictly monotone maps with a finite number of pieces of monotonicity (upon replacing $K(f)$ by the set of turning points of $f$ ). We have just chosen the more restrictive setting of $D(I)$ to simplify the notation (notice that if $f \in D(I)$ then $K(f)$ may be larger than the set of turning points of $f$ ).

Let $\left(\mathcal{B}^{n}\right)_{n=1}^{\infty}$ be the basic covering of $A$. Set $\mathcal{B}^{n}=\left(I_{i}^{n}\right)_{i=0}^{k_{n}-1}$ and $B_{n}=$ $\bigcup_{i=0}^{k_{n}-1} I_{i}^{n}$. By (3) and the fact that $f^{k_{n+1}}\left(I_{0}^{n+1}\right) \subset I_{0}^{n+1}$, there is a periodic orbit $P_{n}$ of period $k_{n+1}$ having exactly one point in each of the intervals $I_{i}^{n+1}$. Then we can find a strongly invariant set $Q_{n}$ with $\operatorname{Card} Q_{n}=k_{n+1}-1$ and satisfying the condition from Theorem A with respect to $P_{n}$. Observe that if $x \in Q_{n} \cap B_{n}$ the orbit of $x$ is also contained in $B_{n}$. This means that $R_{n}=$ $Q_{n} \cap B_{n}$ is a strongly invariant set. On the other hand, Card $Q_{n}=k_{n+1}-1$ implies that $Q_{n} \cap B_{n+1}=\emptyset$. Thus Card $R_{n}=k_{n+1}-k_{n}$ and if $J$ is one of the $k_{n+1}-k_{n}$ (connected) components of $B_{n} \backslash B_{n+1}$, then $\operatorname{Card}\left(R_{n} \cap J\right)=1$ (see (4)). Finally, notice that since the covering is basic, $K(f) \cap B_{1}=K(f) \cap A$ and then $f^{\prime}(x)$ exists for any $x \in R_{n}$.

Now it is the time to use the specific features of piecewise linear maps. Assume additionally $f \in L(I)$ and write $\delta_{n}=\prod_{x \in R_{n}}\left|f^{\prime}(x)\right|$. Then

$$
\delta_{n}>1 \text {. }
$$

To prove this, let $x \in R_{n}$ and suppose that the period of $x$ is $l$. It is sufficient to show that $\left|\left(f^{l}\right)^{\prime}(x)\right|>1$. Suppose $\left|\left(f^{l}\right)^{\prime}(x)\right| \leq 1$ and let $r$ be such that $x \in I_{r}^{n}$. Let $g$ be the restriction of $f^{l}$ to the interval $I_{r}^{n}$. Since $k_{n}$ divides $l$ and the restriction $h$ of $f^{k_{n}}$ to $I_{r}^{n}$ is not monotone, $g=h^{l / k_{n}}$ cannot be linear. Let $K \subset I_{r}^{n}$ be the maximal closed interval containing $x$ on which $g$ is linear. Then at least one of the endpoints of $K$ is an asymptotically periodic point of $g$ and so an asymptotically periodic point of $f$. Let $e$ be such an endpoint of $K$. We may assume that $e$ is not an endpoint of $I_{r}^{n}$ since otherwise the other endpoint $e^{\prime}$ of $K$ is also asymptotically periodic for $g$ and $e^{\prime}$ cannot be an endpoint of $I_{r}^{n}$ (because $\left.K \neq I_{r}^{n}\right)$. Then $g^{\prime}(e)$ does not exist. This means that $f^{m}(e) \in K(f)$ for some $m \in\{0,1, \ldots, l-1\}$. But then $f^{m}(e) \in B_{n} \cap K(f)$ and so $f^{m}(e) \in A$. Since $f^{m}(e)$ is asymptotically periodic we get a contradiction by (2). This proves (5). 
Another important property of the sets $R_{n}$ is the following. Let $G$ be an arbitrary set containing exactly one point from each interval from $\mathcal{B}^{n+1}$ and such that $G \cap K(f)=\emptyset$. Since each interval from $\mathcal{B}^{1}$ can contain at most one point from $K(f)$, clearly there exists a (not necessarily unique) set $H \subset G$ containing exactly one point from each interval from $\mathcal{B}^{1}$ such that if we write

$$
G \backslash H=\left\{a_{1}, \ldots, a_{k_{n+1}-k_{1}}\right\}, \quad \bigcup_{r=1}^{n} R_{r}=\left\{b_{1}, \ldots, b_{k_{n+1}-k_{1}}\right\},
$$

and $I_{i}=\left[a_{i} ; b_{i}\right]$ for $i=1, \ldots, k_{n+1}-k_{1}$, then

$$
\begin{gathered}
I_{i} \cap I_{j}=\emptyset \quad \text { for any } 1 \leq i<j \leq k_{n+1}-k_{1}, \\
\bigcup_{i=1}^{k_{n+1}-k_{1}} I_{i} \subset B_{1} \backslash K(f) .
\end{gathered}
$$

(At this moment we do not need (6) but we shall use it in the proof of Theorem C.) Note that, since $f$ is piecewise linear, (7) implies that $f^{\prime}\left(a_{i}\right)=$ $f^{\prime}\left(b_{i}\right)$ for every $i=1, \ldots, k_{n+1}-k_{1}$.

We are now ready to prove Theorem B.

Proof of Theorem B. Suppose that $A$ is a weakly bounded solenoid of $f$. Since $f$ belongs to $L(I)$ it has no wandering intervals and, hence, $A$ has a basic covering $\left(\mathcal{B}^{n}\right)_{n=1}^{\infty}$. Let $\left(k_{n}\right)_{n=1}^{\infty}$ be the type of $\left(\mathcal{B}^{n}\right)_{n=1}^{\infty}$. Since $A$ is weakly bounded and the covering is basic we see that there exist $l_{0} \in \mathbb{N}$ and a strictly increasing sequence $\left(n_{j}\right)_{j=1}^{\infty}$ such that $k_{n_{j}+1} / k_{n_{j}}=l_{0}$ for any $j$. We claim that there is $\delta>1$ such that

$$
\delta_{n_{j}} \geq \delta \quad \text { for any } j .
$$

To prove this, let $L$ be the (finite) set of all possible values of $\left|f^{\prime}\right|$ (where it is defined) and define $M=\left\{\prod_{m=1}^{k_{1}\left(l_{0}-1\right)} \varrho_{m}: \varrho_{m} \in L\right.$ for any $\left.m\right\}$, also a finite set. Notice that $R_{n_{j}}$ intersects each interval from $\mathcal{B}^{n_{j}}$ in exactly $l_{0}-1$ points. Therefore, we can decompose $R_{n_{j}}$ in $l_{0}-1$ pairwise disjoint finite sets $G_{1}, \ldots, G_{l_{0}-1}$ such that each of the sets $G_{i}$ has exactly one point in each interval from $\mathcal{B}^{n_{j}}$. Then for each $s \in\left\{1, \ldots, l_{0}-1\right\}$ there exists a set $H_{s} \subset G_{s}$ containing exactly one point in each interval from $\mathcal{B}^{1}$ such that, if we write

$$
G_{s} \backslash H_{s}=\left\{a_{1}^{s}, \ldots, a_{k_{n_{j}}-k_{1}}^{s}\right\}, \quad \bigcup_{r=1}^{n_{j}-1} R_{r}=\left\{b_{1}, \ldots, b_{k_{n_{j}}-k_{1}}\right\},
$$

then $f^{\prime}\left(a_{i}^{s}\right)=f^{\prime}\left(b_{i}\right)$ for any $i$ (here we are using property (7) applied to $G_{s}$, $H_{s}$ and $n_{j}$ instead of $G, H$ and $n+1$ for each $\left.s=1, \ldots, l_{0}-1\right)$. Therefore, 


$$
\prod_{x \in G_{s} \backslash H_{s}}\left|f^{\prime}(x)\right|=\prod_{i=1}^{k_{n_{j}}-k_{1}}\left|f^{\prime}\left(a_{i}^{s}\right)\right|=\prod_{n=1}^{n_{j}-1} \delta_{n}
$$

for each $s$. Consequently,

$$
\begin{aligned}
\delta_{n_{j}} & =\prod_{x \in R_{n_{j}}}\left|f^{\prime}(x)\right|=\left(\prod_{x \in \bigcup_{s=1}^{l_{0}-1} H_{s}}\left|f^{\prime}(x)\right|\right)\left(\prod_{x \in \bigcup_{s=1}^{l_{0}-1} G_{s} \backslash H_{s}}\left|f^{\prime}(x)\right|\right) \\
& =\nu_{j}\left(\prod_{n=1}^{n_{j}-1} \delta_{n}\right)^{l_{0}-1}
\end{aligned}
$$

for some $\nu_{j} \in M$. Since the set $\left\{\nu_{j}\right\}_{j=1}^{\infty}$ is finite, we can find $t$ such that for any $j>t$ there is $j^{\prime} \leq t$ with $\nu_{j}=\nu_{j^{\prime}}$. Then, by (5), for any $j>t$ we have

$$
\delta_{n_{j}}=\nu_{j}\left(\prod_{n=1}^{n_{j}-1} \delta_{n}\right)^{l_{0}-1}>\nu_{j}\left(\prod_{n=1}^{n_{j^{\prime}}-1} \delta_{n}\right)^{l_{0}-1}=\nu_{j^{\prime}}\left(\prod_{n=1}^{n_{j^{\prime}}-1} \delta_{n}\right)^{l_{0}-1}=\delta_{n_{j^{\prime}}} .
$$

Thus (8) holds for $\delta=\min _{1 \leq j \leq t} \delta_{n_{j}}$.

Similarly, define $N=\left\{\prod_{m=1}^{k_{1}} \varrho_{m}: \varrho_{m} \in L\right.$ for any $\left.m\right\}$ and take $J \in$ $\mathcal{B}^{n+1}$ and $x \in J$ such that $\left\{f^{j}(x)\right\}_{j=0}^{k_{n+1}-1}$ does not intersect $K(f)$. By using (7) as above we see that there is $\varrho \in N$ (depending on $x$ ) such that

$$
\left|\left(f^{k_{n+1}}\right)^{\prime}(x)\right|=\varrho \prod_{r=1}^{n} \delta_{r}
$$

(here we take the set $\left\{f^{j}(x)\right\}_{j=0}^{k_{n+1}-1}$ for $G$ ). On the other hand, since each of the intervals from $\mathcal{B}^{1}$ contains at most one point from $K(f)$, clearly there exists a positive integer $u$ depending neither on $n$ nor on $J$ such that if $g$ is the restriction of $f^{k_{n+1}}$ to $J$ then Card $K(g)<u$ for any $J \in \mathcal{B}^{n+1}$. In other words, $\left|g^{\prime}\right| \leq u$ whenever it is defined. But if $n$ tends to infinity then from (5), (8) and (9) we deduce that $\left|g^{\prime}\right|$ tends uniformly to infinity; a contradiction.

The proof of Theorem $\mathrm{C}$ follows after a sequence of lemmas. But first we need to reformulate (5)-(7) in our new situation. Recall that if $f \in \operatorname{Lp}(I)$ then it has no wandering intervals. So, for a given solenoid $A$ of $f$ we can construct the strongly invariant sets $R_{n}$ as above (we maintain the same notation). The problem now is that if $x \in R_{n}$ is periodic of period $l$ then it is not necessarily true that $\left|\left(f^{l}\right)^{\prime}(x)\right|>1$ or even that $\left|\left(f^{l}\right)^{\prime}(x)\right| \geq 1$. Then we construct two sets $U_{n}$ and $V_{n}$ with the same cardinality as $R_{n}$ (although not necessarily strongly invariant) as follows.

Fix a point in each of the periodic orbits included in $R_{n}$. Let $x$ be one of these points, say of period $l$. Now two possibilities can occur. If $\left|\left(f^{l}\right)^{\prime}(x)\right| \geq 1$ we include $\left\{f^{j}(x)\right\}_{j=0}^{l-1}$ both in $U_{n}$ and $V_{n}$. Otherwise, let $K$ be the maximal 
open interval containing $x$ with the property $\lim _{m \rightarrow \infty}\left|f^{l m}(y)-f^{l m}(x)\right|=0$ for any $y \in K$ (the so-called immediate basin of attraction of $x$ ). Because of (2), neither $K$ nor any of its iterates intersects $B_{n} \backslash B_{n+1}$. So, $f^{l}(K)=K$ and $f^{l}$ is monotone on $K$. If $y<z$ are the endpoints of $K$ then we have either (i) $f^{l}(y)=y, f^{l}(z)=z$ and $\left|\left(f^{l}\right)^{\prime}(y)\right| \geq 1$, or (ii) $f^{l}(y)=z, f^{l}(z)=y$ and $\left|\left(f^{2 l}\right)^{\prime}(y)\right| \geq 1$. Now we take $\left\{f^{j}(y)\right\}_{j=0}^{l-1} \subset U_{n},\left\{f^{j}(y)\right\}_{j=0}^{l-1} \subset V_{n}$ if (i) holds, and $\left\{f^{j}(y)\right\}_{j=0}^{l-1} \subset U_{n},\left\{f^{j}(y)\right\}_{j=l}^{2 l-1} \subset V_{n}$ if (ii) holds. So we have $\operatorname{Card} U_{n}=$ $\operatorname{Card} V_{n}=\operatorname{Card} R_{n}=k_{n+1}-k_{n}$ and $\operatorname{Card}\left(U_{n} \cap J\right)=\operatorname{Card}\left(V_{n} \cap J\right)=1$ for any component $J$ of $B_{n} \backslash B_{n+1}$. Write now

$$
\delta_{n}=\sqrt{\prod_{x \in U_{n}}\left|f^{\prime}(x)\right| \cdot \prod_{x^{\prime} \in V_{n}}\left|f^{\prime}\left(x^{\prime}\right)\right| .}
$$

Notice that if $f$ is piecewise linear then $U_{n}=V_{n}=R_{n}$ and $\delta_{n}$ is the number we defined above.

We clearly have

$$
\delta_{n} \geq 1
$$

With regard to (6) and (7), if $G$ and $H$ are defined as above and we set

$$
\bigcup_{r=1}^{n} U_{r}=\left\{c_{1}, \ldots, c_{k_{n+1}-k_{1}}\right\}, \quad \bigcup_{r=1}^{n} V_{r}=\left\{d_{1}, \ldots, d_{k_{n+1}-k_{1}}\right\}
$$

$L_{i}=\left[a_{i} ; c_{i}\right]$ and $M_{i}=\left[a_{i} ; d_{i}\right]$ for $i=1, \ldots, k_{n+1}-k_{1}$, then similarly we get

(6b) $\quad L_{i} \cap L_{j}=\emptyset, \quad M_{i} \cap M_{j}=\emptyset \quad$ for any $1 \leq i<j \leq k_{n+1}-k_{1}$,

$$
\bigcup_{i=1}^{k_{n+1}-k_{1}}\left(L_{i} \cup M_{i}\right) \subset B_{1} \backslash K(f) .
$$

In what follows $\lambda$ will denote the one-dimensional Lebesgue measure. A family $\left\{I_{j}\right\}_{j=1}^{m}$ of subintervals of $I$ is said to have multiplicity $k$ if each point from $I$ is contained in at most $k$ intervals from the family. The proof of the first lemma is trivial and therefore omitted.

Lemma 1. Let $\left\{I_{j}\right\}_{j=1}^{m}$ be a family of subintervals of I with multiplicity $k$. Then $\sum_{j=1}^{m} \lambda\left(I_{j}\right) \leq k \lambda\left(\bigcup_{j=1}^{m} I_{j}\right)$.

LEMMA 2. Let $f \in C(I)$ and let $p$ be a periodic point of $f$ of period $n$. Let $1 \leq l \leq n$ and let $J$ be a subinterval of I containing $p$. Then $\left\{f^{j}(J)\right\}_{j=0}^{l-1}$ has multiplicity at most $2 \operatorname{Card}\left(\operatorname{Orb}_{f}(p) \cap f^{l}(J)\right)$.

Proof. Let $m$ be the multiplicity of $\left\{f^{j}(J)\right\}_{j=0}^{l-1}$ and fix a point $x$ belonging to $m$ intervals from the family, say $f^{j_{1}}(J), \ldots, f^{j_{m}}(J)$. If $P$ is the set of points from $\operatorname{Orb}_{f}(p)$ contained in the union of these $m$ intervals then Card $P \geq m$. Let $a, b$ denote the endpoints of Conv $P$. We can assume that $[a ; x]$ contains at least $m / 2$ points from $P$. On the other hand, 
the point $a$ belongs to one of the intervals from the family, say $f^{j_{i}}(J)$. Therefore, $\operatorname{Card}\left(P \cap f^{j_{i}}(J)\right) \geq m / 2$. So, since $P \subset \operatorname{Orb}_{f}(p)$ we also get $\operatorname{Card}\left(\operatorname{Orb}_{f}(p) \cap f^{l}(J)\right) \geq \operatorname{Card}\left(f^{l-j_{i}}(P) \cap f^{l}(J)\right) \geq m / 2$ and the lemma follows.

If $f \in \operatorname{Lp}(I)$ then $\log \left|f^{\prime}\right|$ can clearly be extended to a (not necessarily continuous) map $h$ from $I$ into the real line with minimal bounded variation. The total variation of this map $h$ will be denoted by $V(f)$. Also, in what follows we fix a constant $\alpha(f)>0$ (depending only on $f$ ) such that $|\log | f^{\prime}(x)|-\log | f^{\prime}(y)|| \leq \alpha(f)|x-y|$ for any $x, y \in I$ with $[x ; y] \cap K(f)=\emptyset$.

Lemma 3. Let $f \in \operatorname{Lp}(I), J$ be a subinterval of $I, l \in \mathbb{N}, k$ be the multiplicity of $\left\{f^{j}(J)\right\}_{j=0}^{l-1}$ and let $x, y \in J$ be such that $f^{j}(x), f^{j}(y) \in$ $I \backslash K(f)$ for any $j=0,1, \ldots, l-1$. Then

$$
\frac{\left|\left(f^{l}\right)^{\prime}(x)\right|}{\left|\left(f^{l}\right)^{\prime}(y)\right|} \leq \exp \left(k \alpha(f) \lambda\left(\bigcup_{j=0}^{l-1} f^{j}(J)\right)+k V(f) \operatorname{Card} K(f)\right) .
$$

Moreover, if $f^{j}(J) \cap K(f)=\emptyset$ for any $j=0,1, \ldots, l-1$ then

$$
\frac{\left|\left(f^{l}\right)^{\prime}(x)\right|}{\left|\left(f^{l}\right)^{\prime}(y)\right|} \leq \exp \left(k \alpha(f) \lambda\left(\bigcup_{j=0}^{l-1} f^{j}(J)\right)\right) .
$$

Proof. Let $u, v \in I \backslash K(f)$. If $[u ; v] \cap K(f)=\emptyset$ we have $|\log | f^{\prime}(u) \mid-$ $\log \left|f^{\prime}(v)\right||\leq \alpha(f)| v-u \mid$ while if $[u ; v] \cap K(f) \neq \emptyset$ then $|\log | f^{\prime}(u) \mid-$ $\log \mid f^{\prime}(v) \| \leq V(f)$. Further, since $\left\{f^{j}(J)\right\}_{j=0}^{l-1}$ has multiplicity $k$, there are at most $k$ Card $K(f)$ intervals from the family $\left\{f^{j}(J)\right\}_{j=0}^{l-1}$ intersecting $K(f)$. Also notice that

$$
\frac{\left|\left(f^{l}\right)^{\prime}(x)\right|}{\left|\left(f^{l}\right)^{\prime}(y)\right|} \leq \exp \left(\sum_{j=0}^{l-1}|\log | f^{\prime}\left(f^{j}(x)\right)|-\log | f^{\prime}\left(f^{j}(y)\right)||\right) .
$$

From this and Lemma 1, Lemma 3 follows easily.

Although the following lemma is just a direct consequence of Lemma 3, it is convenient to state it separately.

In what follows we use the following notation. For $f \in \operatorname{Lp}(I)$ and $k \in \mathbb{N}$ define

$$
Q(k, f)=(\operatorname{Card} K(f)+1)^{k \operatorname{Card} K(f)} .
$$

Lemma 4. Let $f \in \operatorname{Lp}(I), J$ be a subinterval of $I, l \in \mathbb{N}, k$ be the multiplicity of $\left\{f^{j}(J)\right\}_{j=0}^{l-1}$, and let $X, Y \subset J$ be measurable sets of positive measure. Then

$$
\frac{\lambda\left(f^{l}(X)\right) / \lambda(X)}{\lambda\left(f^{l}(Y)\right) / \lambda(Y)} \leq Q(k, f) \exp \left(k \alpha(f) \lambda\left(\bigcup_{j=0}^{l-1} f^{j}(J)\right)+k V(f) \operatorname{Card} K(f)\right) .
$$


Proof. For $j=0,1, \ldots, l-1$ denote by $g_{j}$ the restriction of $f$ to the interval $f^{j}(J)$. Further, let $G_{0}$ be the identity map on $J$ and for $j=1, \ldots$, $l-1$ denote by $G_{j}=g_{j-1} \circ \ldots \circ g_{1} \circ g_{0}$ the restriction of $f^{j}$ to $J$. Since $\left\{f^{j}(J)\right\}_{j=0}^{l-1}$ has multiplicity $k$, there are at most $k$ Card $K(f)$ intervals from $\left\{f^{j}(J)\right\}_{j=0}^{l-1}$ intersecting $K(f)$. This means that there is $s \leq k \operatorname{Card} K(f)$ such that for some $0 \leq j(1)<\ldots<j(s) \leq l-1$, the maps $g_{j(i)}, i=1, \ldots, s$, are not monotone and all the other maps $g_{j}$ are monotone.

So, if $P=\left\{a_{0}, a_{1}, \ldots, a_{n}\right\}$ is the set consisting of the endpoints of $J$ and those points $x \in J$ whose finite trajectories $\left(f^{j}(x)\right)_{j=0}^{l-1}$ hit $K(f)$, we have

$$
P=\{\min J, \max J\} \cup \bigcup_{i=1}^{s} G_{j(i)}^{-1}\left(K(f) \cap f^{j(i)}(J)\right) .
$$

Since $f$ is piecewise monotone with Card $K(f)+1$ pieces, all the above implies that

$$
\begin{aligned}
n+1= & \operatorname{Card} P \\
\leq & 2+\operatorname{Card} K(f)+(\operatorname{Card} K(f)+1) \cdot \operatorname{Card} K(f) \\
& +(\operatorname{Card} K(f)+1)^{2} \cdot \operatorname{Card} K(f) \\
& +\ldots+(\operatorname{Card} K(f)+1)^{s-1} \cdot \operatorname{Card} K(f) \\
= & 1+(\operatorname{Card} K(f)+1)^{s} \leq 1+Q(k, f) .
\end{aligned}
$$

Define $I_{i}=\left[a_{i-1}, a_{i}\right]$ and put $X_{i}=X \cap I_{i}, Y_{i}=Y \cap I_{i}, i=1, \ldots, n$. To simplify the notation we assume that all the sets $X_{i}, Y_{i}$ have positive measure. As will be obvious this is not restrictive. Let $r$ and $s$ be such that

$$
\frac{\lambda\left(f^{l}\left(X_{r}\right)\right)}{\lambda\left(X_{r}\right)}=\max _{1 \leq i \leq n} \frac{\lambda\left(f^{l}\left(X_{i}\right)\right)}{\lambda\left(X_{i}\right)}, \quad \frac{\lambda\left(f^{l}\left(Y_{s}\right)\right)}{\lambda\left(Y_{s}\right)}=\min _{1 \leq i \leq n} \frac{\lambda\left(f^{l}\left(Y_{i}\right)\right)}{\lambda\left(Y_{i}\right)},
$$

and fix points $x, y \in J \backslash P$ with the properties

Then

$$
\left|\left(f^{l}\right)^{\prime}(x)\right| \geq \frac{\lambda\left(f^{l}\left(X_{r}\right)\right)}{\lambda\left(X_{r}\right)}, \quad\left|\left(f^{l}\right)^{\prime}(y)\right| \leq \frac{\lambda\left(f^{l}\left(Y_{s}\right)\right)}{\lambda\left(Y_{s}\right)} .
$$

$$
\begin{aligned}
& \frac{\lambda\left(f^{l}(X)\right)}{\lambda(X)} \leq \frac{\sum_{i=1}^{n} \lambda\left(f^{l}\left(X_{i}\right)\right)}{\sum_{i=1}^{n} \lambda\left(X_{i}\right)} \leq \frac{\lambda\left(f^{l}\left(X_{r}\right)\right)}{\lambda\left(X_{r}\right)} \leq\left|\left(f^{l}\right)^{\prime}(x)\right|, \\
& \frac{\lambda\left(f^{l}(Y)\right)}{\lambda(Y)} \geq \frac{1}{n} \frac{\sum_{i=1}^{n} \lambda\left(f^{l}\left(Y_{i}\right)\right)}{\sum_{i=1}^{n} \lambda\left(Y_{i}\right)} \geq \frac{1}{n} \frac{\lambda\left(f^{l}\left(Y_{s}\right)\right)}{\lambda\left(Y_{s}\right)} \geq \frac{\left|\left(f^{l}\right)^{\prime}(y)\right|}{n} .
\end{aligned}
$$

By Lemma 3, the lemma follows.

In what follows we say that a sequence $\left(\beta_{n}\right)_{n=1}^{\infty}$ is a tail of a sequence $\left(\alpha_{n}\right)_{n=1}^{\infty}$ if there is a nonnegative $k$ such that $\beta_{n}=\alpha_{n+k}$ for every $n$. If $k$ is positive then the tail is said to be nontrivial.

Lemma 5. Let $f \in \operatorname{Lp}(I)$ and $A$ be a solenoid of $f$. Then $\lambda(A)=0$. 
Proof. The proof follows mutatis mutandis the one from [2]. There it is proved that solenoids of $C^{\infty}$ maps with nonflat critical points have measure zero. The only difference is that we have to use Lemmas 3 and 4 instead of Lemmas $1 \mathrm{a}, 1 \mathrm{~b}$ and 2 of [2]. For completeness we give here a shorter proof (still following the essential ideas from [2]).

Let $\left(\mathcal{B}^{n}\right)_{n=1}^{\infty}$ be the basic covering of $A$. Fix an interval $I^{\prime}=[a, b]$ with $I \subset(a, b)$ and let $g \in C\left(I^{\prime}\right)$ be defined by the following properties:

(i) $g$ coincides with $f$ in the intervals from $\mathcal{B}^{1}$;

(ii) $g$ is linear on the maximal intervals lying between consecutive intervals from $\mathcal{B}^{1}$ (and on the intervals connecting respectively $a$ and the first interval from $\mathcal{B}^{1}$ and $b$ and the last interval from $\mathcal{B}^{1}$ );

(iii) $g(a)=a, g(b)=b$.

Notice that the definition of $\mathcal{B}^{1}$ implies that $g$ cannot have constant linear pieces and so $g \in \operatorname{Lp}\left(I^{\prime}\right)$. Of course $A$ is also a solenoid of $g$. Further, $K(g) \subset A$. Notice that $\left(\mathcal{B}^{n}\right)_{n=1}^{\infty}$ is now a strict covering of $A$ with respect to $g$ but not necessarily its basic covering. In fact, it may happen that an endpoint of an interval from $\mathcal{B}^{1}$ belongs to $K(g) \backslash K(f)$. Then the basic covering of $A$ with respect to $g$ is a tail of $\left(\mathcal{B}^{n}\right)_{n=1}^{\infty}$. If such an endpoint does not exist then still $\left(\mathcal{B}^{n}\right)_{n=1}^{\infty}$ may not be the basic covering of $A$ with respect to $g$ since it may happen that some gaps between the intervals from $\mathcal{B}^{1}$ contain points from $K(f)$ but do not contain any points from $K(g)$. But then it may happen (it depends on which gaps have that property) that $\left(\mathcal{B}^{n}\right)_{n=1}^{\infty}$ is a nontrivial tail of the basic covering of $A$ with respect to $g$. In either case there is a tail of $\left(\mathcal{B}^{n}\right)_{n=1}^{\infty}$ which is simultaneously a tail of the basic covering of $A$ with respect to $g$. Let $\left(\mathcal{C}^{n}\right)_{n=1}^{\infty}$ with $\mathcal{C}^{n}=\left(I_{i}^{n}\right)_{i=0}^{k_{n}-1}$ be such a covering of $A$. For any $n$ and $i \in\left\{0,1, \ldots, k_{n}-1\right\}$, let $J_{i}^{n}$ denote the maximal interval containing $I_{i}^{n}$ such that $g^{k_{n}-i}\left(J_{i}^{n}\right) \subset I_{0}^{n}$. It is not restrictive to assume that there is a (relative) extremum $c$ of $g$ such that $g(c) \in I_{0}^{n}$ for any $n$. Then

$$
c \in J_{k_{n}-1}^{n} \quad \text { for any } n \text {. }
$$

Write $\mathcal{D}^{n}=\left(J_{i}^{n}\right)_{i=0}^{k_{n}-1}, D_{n}=\bigcup_{i=0}^{k_{n}-1} J_{i}^{n}$. We emphasize that $\left(\mathcal{D}^{n}\right)_{n=1}^{\infty}$ need not be a covering of $A$ because $D_{n+1} \subset D_{n}$ does not necessarily hold. If $J_{i}^{n}$ contains an extremum of $g$ then $g\left(J_{i}^{n}\right)$ may not be equal to $J_{i+1}^{n}$ but the inclusion $g\left(J_{i}^{n}\right) \subset J_{i+1}^{n}$ still holds for any $i$ (here $J_{k_{n}}^{n}=J_{0}^{n}$ ) and the intervals from $\mathcal{D}^{n}$ are pairwise disjoint (also $g^{r}\left(J_{1}\right) \cap g^{r}\left(J_{2}\right)=\emptyset$ whenever $J_{1}, J_{2} \in \mathcal{D}^{n}, J_{1} \neq J_{2}$ and $\left.r \geq 0\right)$. This and $K(g) \subset A$ imply that $I_{i}^{n}$ and $J_{i}^{n}$ must contain the same points from $K(g)$ (at most one of them) for any $i$. Moreover, $D_{n} \subset \operatorname{Int} I^{\prime}$ by property (iii) of $g$. Thus

(11) if $J_{i}^{n}$ contains an extremum of $g$ then its two endpoints have the same image, 
while if $J_{i}^{n}$ does not contain any extrema of $g$ then $g\left(J_{i}^{n}\right)=J_{i+1}^{n}$.

Define

$$
\mu=Q(1, f) \exp \left(\alpha(f) \lambda\left(I^{\prime}\right)+V(g) \operatorname{Card} K(g)\right) .
$$

Since $\mu>2$,

$$
\sigma=1-\frac{1}{\mu}\left(\frac{1}{\mu+1}-\frac{1}{4 \mu^{2}}\right)+\frac{1}{4 \mu^{2}}<1 .
$$

Because of $A \subset D_{n}$ for any $n$, to prove the lemma it suffices to show that for any $n$ there is $l$ such that $\lambda\left(D_{l}\right)<\sigma \lambda\left(D_{n}\right)$.

Since $g$ has no wandering intervals, the lengths of the intervals from $\mathcal{D}^{n}$ tend uniformly to 0 as $n$ tends to $\infty$. Thus, for fixed $n$, choose $l$ large enough so that

$$
\sup _{0 \leq j \leq k_{l}-1} \lambda\left(J_{j}^{l}\right)<\frac{1}{4 \mu^{2}} \inf _{0 \leq i \leq k_{n}-1} \lambda\left(J_{i}^{n}\right) .
$$

For any $J \in \mathcal{D}^{n}$, let $X_{J}$ (resp. $Y_{J}$ ) denote the union of all the intervals from $\mathcal{D}^{l}$ intersecting $J$ (resp. included in $J$ ). Since both endpoints of $J$ are eventually mapped into the same point (use (11) and (12)), it is not possible that two intervals from $\mathcal{D}^{l}$ intersect $J$ and not be included in $J$. Therefore either $X_{J}=Y_{J}$ or $X_{J} \backslash Y_{J}$ is an interval from $\mathcal{D}^{l}$. If $J, J^{\prime} \in \mathcal{D}^{n}$ are different, it is clear that $X_{J} \cap X_{J^{\prime}}=\emptyset$. Thus, it only remains to show that $\lambda\left(X_{J}\right)<\sigma \lambda(J)$ for any $J \in \mathcal{D}^{n}$.

Two possibilities can occur. First assume that $J$ contains an extremum $d$ of $g$ and let $K \in \mathcal{D}^{l}$ be the interval containing $d$. By (11) we clearly get $K \subset$ $J$. Moreover, there exists a set $Z_{J} \subset J$ disjoint from $Y_{J} \backslash K$ and satisfying $g\left(Z_{J}\right)=g\left(Y_{J} \backslash K\right)$. By Lemma 4 (clearly, $\left\{g^{j}(J)\right\}_{j=0}^{0}$ has multiplicity 1 ) we get $\lambda\left(Y_{J} \backslash K\right) \leq \mu \lambda\left(Z_{J}\right)$, which together with $\lambda\left(Y_{J} \backslash K\right)+\lambda\left(Z_{J}\right)<\lambda(J)$ gives

$$
\lambda\left(Y_{J} \backslash K\right)<\left(1-\frac{1}{\mu+1}\right) \lambda(J) .
$$

From this and (13) (use also $\mu>2$ ),

$$
\begin{aligned}
& \lambda\left(Y_{J}\right)=\lambda\left(Y_{J} \backslash K\right)+\lambda(K)<\left(1-\frac{1}{\mu+1}+\frac{1}{4 \mu^{2}}\right) \lambda(J), \\
& \lambda\left(X_{J}\right)=\lambda\left(X_{J} \backslash Y_{J}\right)+\lambda\left(Y_{J}\right)<\left(1-\frac{1}{\mu+1}+\frac{1}{2 \mu^{2}}\right) \lambda(J)<\sigma \lambda(J) .
\end{aligned}
$$

Suppose now that $J$ does not contain any extrema of $g$. Let $k$ be minimal such that $g^{k}(J)=J^{\prime} \in \mathcal{D}^{n}$ contains an extremum of $g$ (by (10)-(12) such a $k$ exists). Observe that $g^{k}\left(J \backslash Y_{J}\right)=J^{\prime} \backslash Y_{J^{\prime}}$ by (12) applied to the intervals 
from $\mathcal{D}^{l}$. Moreover, $\left\{g^{j}(J)\right\}_{j=0}^{k-1}$ has multiplicity 1 . Then by Lemma 4,

$$
\frac{1-\lambda\left(Y_{J}\right) / \lambda(J)}{1-\lambda\left(Y_{J^{\prime}}\right) / \lambda\left(J^{\prime}\right)}=\frac{\lambda\left(J \backslash Y_{J}\right) / \lambda(J)}{\lambda\left(J^{\prime} \backslash Y_{J^{\prime}}\right) / \lambda\left(J^{\prime}\right)}=\frac{\lambda\left(J^{\prime}\right) / \lambda(J)}{\lambda\left(J^{\prime} \backslash Y_{J^{\prime}}\right) / \lambda\left(J \backslash Y_{J}\right)} \geq \frac{1}{\mu} .
$$

On the other hand, we have already shown that

$$
\frac{\lambda\left(Y_{J^{\prime}}\right)}{\lambda\left(J^{\prime}\right)}<1-\frac{1}{\mu+1}+\frac{1}{4 \mu^{2}} \text {. }
$$

Thus

$$
\lambda\left(Y_{J}\right)<\left(1-\frac{1}{\mu}\left(\frac{1}{\mu+1}-\frac{1}{4 \mu^{2}}\right)\right) \lambda(J) .
$$

This and (13) imply

$$
\lambda\left(X_{J}\right)=\lambda\left(X_{J} \backslash Y_{J}\right)+\lambda\left(Y_{J}\right)<\frac{1}{4 \mu^{2}} \lambda(J)+\lambda\left(Y_{J}\right)<\sigma \lambda(J)
$$

as we wanted to show.

The following lemma was proved in [9] in the particular case of piecewise linear maps (Expansion-Lemma 3.1). Our argument combines the main ideas from Martens and Tresser's proof with the tools developed earlier.

Lemma 6. Let $f \in \operatorname{Lp}(I)$ and let $A$ be a non-period doubling solenoid of $f$ of type $\left(k_{n}\right)_{n=1}^{\infty}$. Then there is $\delta>1$ which does not depend on $n$ such that, for $n$ large enough, we have $\delta_{n} \geq \delta$ whenever $k_{n+1} / k_{n}>2$.

Proof. Let $\left(\mathcal{B}^{n}\right)_{n=1}^{\infty}$ be the basic covering of $A$. Suppose that the statement of the lemma is not true. Then there is $m$ large enough with $k_{m+1} / k_{m}$ $>2$ so that $\beta$ and $\varepsilon$ defined by $\delta_{m}=\exp \beta$ and $\lambda\left(\bigcup_{J \in \mathcal{B}^{m}} J\right)=2 \varepsilon$ are sufficiently small in order that

$$
Q(12, f) \exp (24 \alpha(f) \varepsilon+12 V(f) \operatorname{Card} K(f))<\frac{1}{\exp (25 \alpha(f) \varepsilon+\beta)-1}
$$

(use Lemma 5 and (5b)).

Let $\mathcal{J}$ denote the family of the $k_{m+1}-k_{m}$ components of the set

$$
\left(\bigcup_{J \in \mathcal{B}^{m}} J\right) \backslash\left(\bigcup_{J^{\prime} \in \mathcal{B}^{m+1}} J^{\prime}\right)
$$

Fix $K_{1} \in \mathcal{J}$ and take $x \in U_{m} \cap K_{1}$ (recall that $U_{m}$ and $V_{m}$ were defined right after the proof of Theorem B). Let $l$ or $2 l$ be the period of $x$ depending on whether $x \in V_{m}$ or not. Obviously $f^{l}\left(K_{1}\right) \supset K_{1}$. We claim that $f^{l}\left(K_{1}\right)$ also includes some interval from $\mathcal{J}$ different from $K_{1}$. Suppose that this is not the case and let $J_{1}, J_{2}$ denote the intervals from $\mathcal{B}^{m+1}$ adjacent to $K_{1}$. Since $l<k_{m+1}$ we see that $f^{l}\left(J_{1}\right) \neq J_{1}$. Then we must have $f^{l}\left(J_{1}\right)=J_{2}, f^{l}\left(J_{2}\right)=J_{1}$ and $k_{m+1}=2 l$. Further, $\operatorname{Conv}\left(f^{j}\left(J_{1}\right) \cup f^{j}\left(J_{2}\right)\right)$ includes no intervals from $\mathcal{B}^{m+1}$ apart from $f^{j}\left(J_{1}\right), f^{j}\left(J_{2}\right), j=0,1, \ldots, l-1$. Since the intervals from $\mathcal{J}$ do no intersect $K(f)$, in particular we get 
$f\left(\operatorname{Conv}\left(f^{j}\left(J_{1}\right) \cup f^{j}\left(J_{2}\right)\right)\right)=\operatorname{Conv}\left(f^{j+1}\left(J_{1}\right) \cup f^{j+1}\left(J_{2}\right)\right)$ for any $j$. Moreover, the intervals $\operatorname{Conv}\left(f^{j}\left(J_{1}\right) \cup f^{j}\left(J_{2}\right)\right)$ are pairwise disjoint for $j=0,1, \ldots, l-1$. Hence $\left(\operatorname{Conv}\left(f^{j}\left(J_{1}\right) \cup f^{j}\left(J_{2}\right)\right)\right)_{j=0}^{l-1}$ is a periodic sequence of closed intervals of period $l$ containing $\bigcup_{J \in \mathcal{B}^{m+1}} J$ and contained in $\bigcup_{J \in \mathcal{B}^{m}} J$. Since $l<k_{m+1}$ and $l>k_{m}$ (here we use the fact that $k_{m+1} / k_{m}>2$ and $k_{m+1}=2 l$ ) we obtain a contradiction with the fact that $\left(\mathcal{B}^{n}\right)_{n=1}^{\infty}$ is the basic covering of $A$.

Let $K_{1}^{j} \in \mathcal{J}$ denote the interval containing $\left\{f^{j}(x): j=0,1, \ldots, l-1\right\}$. Since $f$ is monotone on $K_{1}^{j}$ and $f\left(K_{1}^{j}\right) \supset K_{1}^{j+1}$ for any $j$ (here $K_{1}^{l}=K_{1}$ ), there is an interval $T_{1} \subset K_{1}$ containing $x$ and such that $f^{l}\left(T_{1}\right)=K_{1}$. Construct now a minimal interval $K_{1} \supset T \supset T_{1}$ with the property that $f^{l}(T)$ includes an interval $K_{2} \in \mathcal{J}$ different from $K_{1}$. By the above claim, such a $T$ exists. Choose also $T_{2} \subset T$ with $f^{l}\left(T_{2}\right)=K_{2}$. We can additionally assume that $K_{1}$ is an interval of minimal length from $\mathcal{J}$. In particular $\lambda\left(K_{1}\right) \leq$ $\lambda\left(K_{2}\right)$. Now we consider two cases:

First consider the case when $x \notin V_{m}$. The minimality of $T$ implies that $f^{l}(T)$ intersects at most 3 intervals from $\mathcal{J}$ and then contains at most 6 points from $\operatorname{Orb}_{f}(x)$. Hence, $\left\{f^{j}(T)\right\}_{j=0}^{l-1}$ has multiplicity at most 12 by Lemma 2.

Put $x^{\prime}=f^{l}(x)$. Since the intervals $\left\{f^{j}\left(\left[x ; x^{\prime}\right]\right)\right\}_{j=0}^{l-1}$ are pairwise disjoint and do not intersect $K(f)$, by Lemma $3,\left|\left(f^{l}\right)^{\prime}(x)\right| /\left|\left(f^{l}\right)^{\prime}\left(x^{\prime}\right)\right| \leq \exp (2 \alpha(f) \varepsilon)$. On the other hand, $\left|\left(f^{l}\right)^{\prime}(x)\right| \cdot\left|\left(f^{l}\right)^{\prime}\left(x^{\prime}\right)\right|=\left|\left(f^{2 l}\right)^{\prime}(x)\right| \leq \exp (2 \beta)$. Then

$$
\left|\left(f^{l}\right)^{\prime}(x)\right| \leq \exp (\alpha(f) \varepsilon+\beta) .
$$

Notice that $f^{l}\left(T_{1}\right)=K_{1}$ does not intersect any interval from $\mathcal{B}^{m+1}$ and so, for each $j=0,1, \ldots, l-1, f^{j}\left(T_{1}\right)$ does not intersect any interval from $\mathcal{B}^{m+1}$. We also have $x \in T_{1}$. Then by Lemma 3 and (15),

$$
\left|\left(f^{l}\right)^{\prime}(y)\right| \leq \exp (25 \alpha(f) \varepsilon+\beta)
$$

for any $y \in T_{1}$. In particular,

$$
\frac{\lambda\left(f^{l}\left(T_{1}\right)\right)}{\lambda\left(T_{1}\right)} \leq \exp (25 \alpha(f) \varepsilon+\beta) .
$$

Recall that $\lambda\left(K_{1}\right) \leq \lambda\left(K_{2}\right)$ and obviously $T_{1} \cap T_{2}=\emptyset$. Then

$$
\frac{\lambda\left(f^{l}\left(T_{2}\right)\right)}{\lambda\left(T_{2}\right)} \geq \frac{\lambda\left(K_{1}\right)}{\lambda\left(K_{1}\right)-\lambda\left(T_{1}\right)} \geq \frac{\exp (25 \alpha(f) \varepsilon+\beta)}{\exp (25 \alpha(f) \varepsilon+\beta)-1} .
$$

From Lemma 4 and (14), (16) and (17) we get a contradiction.

The case $x \in V_{m}$ is analogous. The only difference is that now $\left\{f^{j}(T)\right\}_{j=0}^{l-1}$ has multiplicity at most 6 and we have directly $\left|\left(f^{l}\right)^{\prime}(x)\right| \leq \exp \beta$. The lemma is proved.

Proof of Theorem $C$. Suppose that $f$ has a non-period doubling solenoid $A$ with basic covering $\left(\mathcal{B}^{n}\right)_{n=1}^{\infty}$ and of type $\left(k_{n}\right)_{n=1}^{\infty}$. Put $\gamma=\sum_{J \in \mathcal{B}^{1}} \lambda(J)$ 
and fix $\varepsilon>0$ small enough such that

$$
\left|f^{\prime}(x)\right|>\varepsilon
$$

for any $x \in I$ for which $f^{\prime}$ exists.

Let $J \in \mathcal{B}^{n+1}$ and let $x \in J$ be such that $G=\left\{f^{j}(x)\right\}_{j=0}^{k_{n+1}-1}$ does not intersect $K(f)$. Construct $H$ and the intervals $L_{i}=\left[a_{i} ; c_{i}\right], M_{i}=\left[a_{i} ; d_{i}\right]$ as in (6b) and (7b). Observe that by (6b),

$$
\sum_{i=1}^{k_{n+1}-k_{1}} \lambda\left(L_{i}\right)<\gamma, \quad \sum_{i=1}^{k_{n+1}-k_{1}} \lambda\left(M_{i}\right)<\gamma
$$

and by $(7 b)$,

$$
\begin{aligned}
& |\log | f^{\prime}\left(a_{i}\right)|-\log | f^{\prime}\left(c_{i}\right)|| \leq \alpha(f) \lambda\left(L_{i}\right), \\
& |\log | f^{\prime}\left(a_{i}\right)|-\log | f^{\prime}\left(d_{i}\right)|| \leq \alpha(f) \lambda\left(M_{i}\right)
\end{aligned}
$$

for any $i$. Thus

$$
\prod_{i=1}^{k_{n+1}-k_{1}}\left|f^{\prime}\left(a_{i}\right)\right|>\exp (-\alpha(f) \gamma) \prod_{i=1}^{k_{n+1}-k_{1}}\left|f^{\prime}\left(c_{i}\right)\right|
$$

and similarly

$$
\prod_{i=1}^{k_{n+1}-k_{1}}\left|f^{\prime}\left(a_{i}\right)\right|>\exp (-\alpha(f) \gamma) \prod_{i=1}^{k_{n+1}-k_{1}}\left|f^{\prime}\left(d_{i}\right)\right| .
$$

From (18)-(20),

$$
\left|\left(f^{k_{n+1}}\right)^{\prime}(x)\right|>\exp (-\alpha(f) \gamma) \varepsilon^{k_{1}} \prod_{r=1}^{n} \delta_{r} .
$$

From this and Lemma 6 we obtain a contradiction similar to the one from the proof of Theorem B.

\section{References}

[1] L. Alsedà, J. Llibre and M. Misiurewicz, Combinatorial Dynamics and Entropy in Dimension One, Adv. Ser. in Nonlinear Dynam. 5, World Sci., Singapore, 1993.

[2] A. M. Blokh and M. Yu. Lyubich, Measure and dimension of solenoidal attractors of one dimensional dynamical systems, Comm. Math. Phys. 127 (1990), 573-583.

[3] J. Bobok and M. Kuchta, Register shifts versus transitive F-cycles for piecewise monotone maps, Real Anal. Exchange 21 (1995/96), 134-146.

[4] R. Galeeva and S. van Strien, Which families of l-modal maps are full?, Trans. Amer. Math. Soc. 348 (1996), 3215-3221.

[5] V. Jiménez López and L'. Snoha, There are no piecewise linear maps of type $2^{\infty}$, ibid. 349 (1997), 1377-1387. 
[6] S. F. Kolyada, Interval maps with zero Schwarzian, in: Functional-Differential Equations and Their Applications, Inst. Math. Ukrain. Acad. Sci., Kiev, 1985, 4757 (in Russian).

[7] L. Lovász and M. D. Plummer, Matching Theory, Akadémiai Kiadó, Budapest, 1986 .

[8] M. Martens, W. de Melo and S. van Strien, Julia-Fatou-Sullivan theory for real one-dimensional dynamics, Acta Math. 168 (1992), 271-318.

[9] M. Martens and C. Tresser, Forcing of periodic orbits for interval maps and renormalization of piecewise affine maps, Proc. Amer. Math. Soc. 124 (1996), 28632870 .

[10] W. de Melo and S. van Strien, One-Dimensional Dynamics, Springer, Berlin, 1993.

[11] M. Misiurewicz, Attracting Cantor set of positive measure for a $C^{\infty}$ map of an interval, Ergodic Theory Dynam. Systems 2 (1982), 405-415.

[12] C. Preston, Iterates of Piecewise Monotone Mappings on an Interval, Lecture Notes in Math. 1347, Springer, Berlin, 1988.

Departament de Matemàtiques Universitat Autònoma de Barcelona 08193 Bellaterra, Barcelona, Spain

E-mail: alseda@mat.uab.es

Department of Mathematics

Faculty of Natural Sciences

Matej Bel University

Tajovského 40

97401 Banská Bystrica, Slovakia

E-mail: snoha@bb.sanet.sk
Departamento de Matemáticas Universidad de Murcia Campus de Espinardo Aptdo. de Correos 4021 30100 Murcia, Spain E-mail: vjimenez@fcu.um.es

Received 13 August 1997;

in revised form 7 February 1998 